Article

\title{
Diversity of Endophytic Fungi in Huperzia serrata and Their Acetylcholinesterase Inhibitory Activity
}

\author{
Ziyun Lu ${ }^{1,2,+}$, Yangshuai Ma ${ }^{2,+}$, Liting Xiao ${ }^{2}$, Huilin Yang ${ }^{1,2,3, *(1)}$ and Du Zhu ${ }^{2}$ \\ 1 Ministry of Education's Key Laboratory of Poyang Lake Wetland and Watershed Research, \\ Jiangxi Normal University, Nanchang 330200, China; luziyun675@outlook.com \\ 2 Key Laboratory of Protection and Utilization of Subtropic Plant Resources of Jiangxi Province, \\ Jiangxi Normal University, Nanchang 330200, China; mayangshuai@outlook.com (Y.M.); \\ xiaolt014726@163.com (L.X.); zhudu12@163.com (D.Z.) \\ 3 Nanchang Key Laboratory of Microbial Resources Exploitation \& Utilization from Poyang Lake Wetland, \\ Jiangxi Normal University, Nanchang 330013, China \\ * Correspondence: yanghl@jxnu.edu.cn \\ + These authors contributed equally to this paper.
}

check for updates

Citation: Lu, Z.; Ma, Y.; Xiao, L.; Yang, H.; Zhu, D. Diversity of Endophytic Fungi in Huperzia serrata and Their Acetylcholinesterase Inhibitory Activity. Sustainability 2021, 13, 12073. https://doi.org/10.3390/ su132112073

Academic Editors: Dario Donno, Emanuele Radicetti and José Manuel Mirás-Avalos

Received: 15 September 2021

Accepted: 26 October 2021

Published: 1 November 2021

Publisher's Note: MDPI stays neutral with regard to jurisdictional claims in published maps and institutional affiliations.

Copyright: (c) 2021 by the authors. Licensee MDPI, Basel, Switzerland. This article is an open access article distributed under the terms and conditions of the Creative Commons Attribution (CC BY) license (https:/ / creativecommons.org/licenses/by/ $4.0 /)$.

\begin{abstract}
Huperzia serrata is a Huperzine A (HupA)-producing herb. HupA is a potent, reversible, highly specific, centrally active and selective acetylcholinesterase inhibitor, and is commonly used to improve Alzheimer's disease.At present, H. serrata resources have been overexploited and their artificial cultivation has lagged behind the need, so the market demand cannot be met. In this study, the diversity of the endophytic fungi in H. serrata was studied by high-throughput sequencing and traditional culture methods. Furthermore, the ability of the isolated endophytic fungi to produce acetylcholinesterase-inhibiting activity was evaluated. The results related to the diversity of fungi between high-throughput sequencing and traditional culture methods were compared. With highthroughput sequencing, five phyla, 22 classes, 55 orders, 120 families, and 178 genera of fungi were detected in Hubei Province, and five phyla, 22 classes, 54 orders, 124 families, and 196 genera were detected in Fujian Province. After cultivation with traditional culture methods, two phyla, three classes, five orders, six families, and six genera were detected in Hubei, and one phylum, three classes, five orders, five families, and six genera in Fujian. The endophytic fungi of $H$. serrata are highly diverse, and the results of high-throughput sequencing comprehensively reflect their community compositions. When the acetylcholinesterase-inhibiting activity of the isolated and cultured endophytic fungi was determined, five and three endophytic fungi from Hubei and Fujian Provinces, respectively, had good inhibitory activity (inhibition rate $>50 \%$ ). Most of them were separated from leaf tissue. The strain HBR-1 and strain FJL-5 had the strongest inhibitory effects on AChE, with inhibition rates of $72.34 \%$ and $60.54 \%$, respectively. Although only a proportion of the endophytic fungi was isolated with traditional culture methods, metabolites with broad potential applications can be isolated from these fungal cultures. The combination of high-throughput sequencing and traditional culture methods can be used to isolate and purify endophytic fungi in a targeted manner, gain many endophytic fungi, and enrich the endophytic fungi resource library.
\end{abstract}

Keywords: acetylcholinesterase inhibitor; diversity; endophytic fungi; high-throughput sequencing; Huperzia serrata; traditional culture method

\section{Introduction}

Huperzia serrata (Thunb. ex Murray) Trev. is a commonly used Chinese herbal medicine. It is a fern belonging to the subfamily Huperzioideae and the genus Huperzia Bemh. The taxonomic status of huperaceae is still partly confused [1]. Huperzia is distributed worldwide (Asia, Oceania, Central America, etc.) and mainly grows in moist forests and rock cracks at altitudes of 300-2700 $\mathrm{m}$ in China [2]. The whole plant has long been used in Chinese medicine to treat various ailments [3], including contusions, strains, schizophrenia, 
myasthenia gravis, abdominal distension, hematuria, and organophosphate poisoning [4] Huperzia plants are rich in alkaloids [5]. Huperzine A (HupA) was the first alkaloid isolated from $H$. serrata, in the 1980s. It is a highly efficient acetylcholinesterase (AChE) inhibitor, with low toxicity and few side effects. It has an extremely significant effect on improving memory and treating early-stage Alzheimer's disease, with less toxicity and side effects than other treatments, and it has broad market potential [6]. H. serrata has attracted intense attention worldwide since its marked anticholinesterase activity was discovered by Chinese scientists. Huperzine A from Huperzia is approved for use as a food additive in the United States [7]. H. serrata is a perennial herb, with slow growth and low biomass, and wild resources are limited. Moreover, this rare wild resource is being driven to extinction by its predatory exploitation, environmental degradation, and other factors.

Plant endophytes are those bacteria, actinomycetes, and fungi that live in various tissues and organs of healthy plants at a certain stage or all stages of their life histories. The infected host generally shows no external symptoms [8-10]. Endophytes can be isolated from plant tissues after strict surface disinfection with histological methods, or their DNA can be directly amplified from the tissues to confirm their endogenesis [11]. Endophytes can promote plant growth by altering the plant physiology, including by regulating osmotic pressure, changing stomata size and morphology, modifying the root metabolism, and increasing the absorption of certain minerals [12]. Endophytes can also produce metabolites, such as glucanase, chitinase [13], hydrolase, and antibiotics, which can be used as biological control agents [14]. Plant endophytes have also been shown to have repair functions and increase plant resistance to stress [15]. Some endophytic fungi produce compounds similar to those produced by their hosts $[16,17]$. Others are potential sources of natural products, such as antibiotics and anticancer agents [18]. Endophytes are recognized as reservoirs of novel compounds of great value in agriculture, industry, and medicine [19].

Studies of the diversity of endophytic fungi have mainly sought to obtain microbial cultures through separation and culturing, and the subsequent identification and classification of the isolated strains. However, given the limitations of experimental conditions, it is impossible to provide the same environments in which microorganisms naturally exist, so large numbers of microorganisms cannot be isolated with traditional methods of separation and culture. However, the rapid development of high-throughput sequencing technology has provided a more effective way to study the diversity of plant endophytic fungi, and can comprehensively and objectively determine the microbial colony structure and the relative compositions of microbial communities in a target environment.

The endophytic fungi of $H$. serrata have mainly been studied with traditional culture methods, and few researchers have used high-throughput sequencing to determine the diversity of this endophytic fungi $[20,21]$. To more comprehensively understand the diversity of endophytic fungi in $H$. serrata, we used high-throughput sequencing and traditional culture methods to study plants from Fujian and Hubei Provinces. We first isolated and identified the endophytic fungi in the roots, stems, and leaves of plants from the two provinces. We then studied the microbial diversity in the roots, stems, and leaves of the plants and the corresponding rhizosphere soil, and compared the differences in the diversity of endophytic fungi determined with high-throughput sequencing and traditional culture methods. We also evaluated the ability of the isolated endophytic fungi to produce AchE inhibitors.

\section{Materials and Methods}

\subsection{Materials}

Healthy H. serrata plants were collected in May 2019, from Hubei $\left(29.93^{\circ} \mathrm{N}, 109.27^{\circ} \mathrm{E}\right)$ and Fujian $\left(27.11^{\circ} \mathrm{N}, 119.28^{\circ} \mathrm{E}\right)$ Provinces, China. All samples were immediately transferred to the laboratory in an icebox. Six plants were collected in total, three samples each in Hubei and Fujian Province. The plants' roots, stems and leaves were screened for endophytic fungi within $24 \mathrm{~h}$. 


\subsection{Sample Processing}

The samples were sterilized according to Beckers et al. [22], with some modification. Briefly, the root samples were shaken and the fallen soil particles removed. The remaining soil was separated from the root tissue as the "rhizosphere soil" compartment. To eliminate any epiphytic microorganisms, the plants were (a) rinsed with sterile water for $10 \mathrm{~min}$, (b) rinsed with $70 \%$ ethanol for $2 \mathrm{~min}$, (c) rinsed three times with sterile water, (d) soaked in sodium hypochlorite solution ( $2.5 \%$ active $\mathrm{Cl}^{-}$with $0.1 \%$ Tween 80 ) for $5 \mathrm{~min}$, (e) rinsed in $70 \%$ ethanol for $1 \mathrm{~min}$, and finally (f) rinsed five times with sterile water. In order to confirm the surface disinfection process was successful, sterility was checked with potato dextrose agar (PDA) [23]. The samples were cut into $0.5 \mathrm{~cm}^{2}$ sections, and some were transferred to a Petri dish containing potato dextrose agar (PDA) or Czapek-Dox medium. The samples were incubated at $28^{\circ} \mathrm{C}$ for 7 days and the endophytic fungi separated according to their different morphological characteristics. Tip mycelia were picked and transferred to a solid medium of PDA or Czapek-Dox medium and incubated at $28^{\circ} \mathrm{C}$. After colony growth, the previous steps were repeated until pure culture was obtained. The fungal isolates were stored in medium slants at $4{ }^{\circ} \mathrm{C}$ or as spore suspensions in $15 \%(\mathrm{v} / \mathrm{v})$ glycerol at $-80{ }^{\circ} \mathrm{C}$.

\subsection{DNA Extraction}

An aliquot $(1.5 \mathrm{~mL})$ of each homogenized plant material was centrifuged $(13,000 \times g$, $10 \mathrm{~min}$ ). The supernatants were discarded, and DNA was extracted from the plant tissues with the E.Z.N.A. HP Plant DNA Mini Kit (Omega, Guangzhou, China), according to the manufacturer's instructions; DNA was extracted from the rhizosphere soil with the FastDNA $^{\mathrm{TM}}$ Spin Kit for Soil (MP Medicals, Shanghai, China), according to the protocol provided by the manufacturer, and DNA was extracted from the isolated endophytic fungi with the E.Z.N.A. ${ }^{\mathrm{TM}}$ HP Fungal DNA Kit (Omega), according to the manufacturer's protocol.

\subsection{PCR Amplification and High-Throughput Sequencing}

The DNA from the rhizosphere soil, roots, stems, and leaves was amplified with optimal primers ITS1F ( $5^{\prime}$-CTTGGTCATTTAGAGGAAGTAA-3 ${ }^{\prime}$ ) and ITS2R (5'-GCTGCGTTCTTCATCGATGC-3') [24] and fungal libraries were created. The PCR products were purified with the E.Z.N.A. ${ }^{\circledR}$ Gel Extraction Kit (Omega). The DNA content was determined with a Qubit 3 fluorometer (Thermo Fisher Scientific Inc., Waltham, MA, USA). The gel extraction products were then mixed in equal amounts. Finally, the PCR products were sequenced (PE250) with the Illumina HiSeq 2500 high-throughput sequencing platform (Novogene Co., Ltd., Beijing, China). The barcode and primer sequences of original data were removed and then spliced. The spliced sequences were strictly filtered and the chimeric sequences removed to obtain the final valid data.

The DNA extracted from the isolated endophytic fungi was amplified with PCR with primers ITS1 (5'-TCCGTAGGTGAACCTGCGG-3') and ITS4 (5'-TCCTCCGCTTATTGATATGC-3'), and then sequenced (Sangon Biotech Co., Ltd., Shanghai, China).

\subsection{Sequence Availability}

The microbiome sequences have been deposited in the National Center for Biotechnology Information (NCBI) database under BioProject accession number PRJNA655213 and under the Sequence Read Archive (SRA) accession numbers SRR12401455-SRR12401478.

\subsection{Diversity Analysis of Endophytic Fungi}

All effective tags of all the samples were clustered with the UCLUST [25], and sequences with $\geq 97 \%$ similarity were assigned to the same operational taxonomic unit (OTU). Rarefaction curves were computed with QIIME (version 1.8.0) [26]. The ITS sequences of the endophytic fungi were compared to the data available in NCBI using BLAST searches to estimate the phylogenetic relationships of the endophytic fungi. A phylogenetic analysis was performed with MEGA version 6.06 [27]. Phylogenetic trees were constructed with the neighbor joining (NJ) method. A principal coordinates analysis (PCoA) and a cluster 
analysis based on the unweighted pair-group method with arithmetic means (UPGMA) were conducted in $\mathrm{R}$ (version 2.15.3). The diversity of the endophytic fungi in $H$. serrata was determined with the Shannon index and Simpson index.

Shannon index:

$$
H=-\sum_{i=1}^{N} P_{i} \ln P_{i}
$$

Simpson index:

$$
D=1-\sum P_{i}^{2}
$$

In the formulae, $N$ represents the sum of the number of individuals of all strains; $\mathrm{Pi}=\mathrm{Ni} / \mathrm{N}$, where $\mathrm{Ni}$ is the number of individuals from the $i$ th genus in the different tissues of the plants, and $N$ is the sum of the number of individual strains of all genera.

\subsection{Fermentation and Preparation of Endophytic Fungal Extracts}

Potato dextrose broth (PDB) liquid medium was inoculated with the individual isolated endophytic fungi. They were cultured at $160 \mathrm{rpm}$ at $28^{\circ} \mathrm{C}$ for 14 days in a rotary shaker. The mycelial pellets were collected by centrifugation at $13,000 \times g$ for $10 \mathrm{~min}$, freezedried, and then ground in a mortar with liquid nitrogen. Each sample of raw material $(1.0 \mathrm{~g})$ was extracted with $75 \%$ ethanol $(50 \mathrm{~mL})$ for $30 \mathrm{~min}$; this was repeated three times and the extracts filtered. The filtrates were combined and evaporated under reduced pressure. The dry residue was dissolved in $50 \mathrm{~mL}$ of $2.5 \%$ hydrochloric acid, and purified by shaking twice with chloroform and then with ethylic ether. The aqueous phase was rendered basic with a $25 \%$ ammonia solution ( $\mathrm{pH} 9$ ), salted out with sodium chloride, and then extracted thoroughly with chloroform. The chloroform-combined extract was evaporated to dryness. The dried residue was dissolved in $10 \mathrm{~mL}$ of (HPLC-grade) methanol, and the methanol extract was filtered through a $0.45 \mu \mathrm{m}$ filter [28].

\subsection{Determination of AchE Inhibitory Activity in Endophytic Fungal Fermentation Products}

The in vitro AchE-inhibiting activities of the endophytic fungal extracts were determined with the method of Atta-ur-Rahman et al. [29], and compared with that of authentic HupA. The preincubation samples contained $250 \mu \mathrm{L}$ of phosphate buffer ( $\mathrm{pH} 7.7$ ), which consisted of $15 \mu \mathrm{L}$ of fungal extract or authentic HupA, $80 \mu \mathrm{L}$ of dithiobis nitrobenzoic acid (DTNB; $3.96 \mathrm{mg}$ DTNB and $1.5 \mathrm{mg}$ sodium bicarbonate dissolved in $10 \mathrm{~mL}$ phosphate buffer (pH 7.7)), and $10 \mu \mathrm{L}$ of $\mathrm{AChE}\left(0.22 \mathrm{U} \cdot \mathrm{mL}^{-1}\right)$. The mixture was incubated at $25^{\circ} \mathrm{C}$ for $5 \mathrm{~min}$. After preincubation, $15 \mu \mathrm{L}$ of the substrate acetylthiocholine iodide $(10.85 \mathrm{mg}$ in $5 \mathrm{~mL}$ of phosphate buffer) was added and incubated again for $5 \mathrm{~min}$. Color development was measured in a 96-well microtiter plate at a wavelength of $412 \mathrm{~nm}$ in an iMark ${ }^{\mathrm{TM}}$ Microplate Absorbance Reader (Bio-Rad, Hercules, CA, USA). Percentage inhibition was calculated with the formula: ([control absorbance - sample absorbance]/control absorbance) $\times 100 \%$.

\section{Results}

3.1. Analysis of Endophytic Fungal Community Structures in H. serrata with High-Throughput Sequencing

3.1.1. Alpha Rarefaction Curves and Alpha Diversity

The abundances of the plant endophytic fungal communities in the samples from both Fujian and Hubei Provinces were lower than that of the corresponding rhizosphere soil flora (Figure 1). The abundance of the endophytic fungi in the roots, stems, and leaves was similar. The rhizosphere soil samples from Hubei contained 648, 652 and 665 operational taxonomic units (OTUs), and those from Fujian contained 668, 674, and 676 operational taxonomic units (OTUs). There was a combined total of 375, 378 and 389 OTUs in the root, 367,371 and 383 OTUs in the stem, and 353, 369 and 377 OTUs in the leaf samples from Hubei, while there was a combined total of 450, 453 and 454 OTUs in the root, 416, 430 and 433 OTUs in the stem, and 497, 516 and 518 in the leaf samples from Fujian. 
a

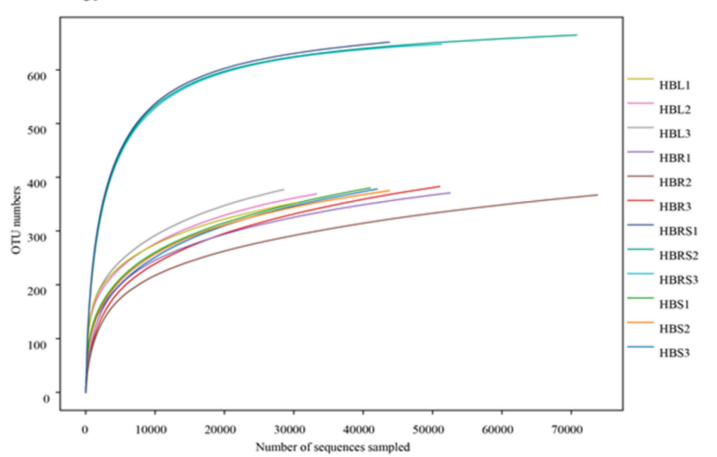

$\mathrm{b}$

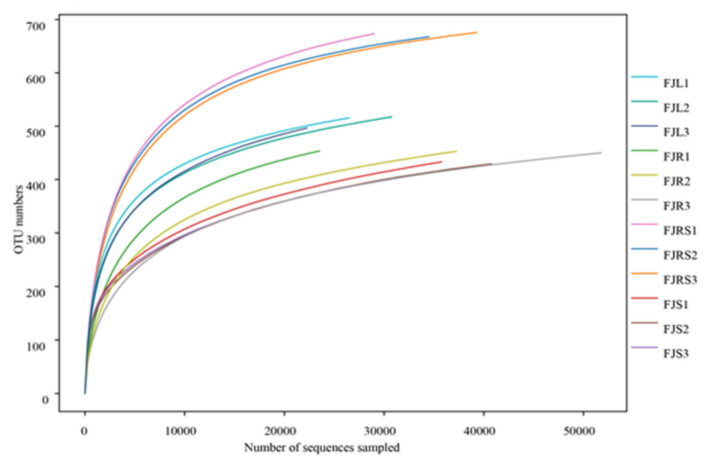

Figure 1. Rarefaction curves of fungi in rhizosphere soil and of endophytic fungi in different tissue. (a) Rarefaction curves of Huperzia serrata samples from Hubei Province. (b) Rarefaction curves of Huperzia serrata samples from Fujian Province. Note: HBL: H. serrata leaf, HBR: H. serrata root, HBS: H. serrata stem, HBRS: rhizosphere soil; FJL: H. serrata leaf, FJR: $H$. serrata root, FJS: H. serrata stem, FJRS: rhizosphere soil. The numbers 1, 2, and 3 in the names are used to indicate that the same tissue sample is repeated three times.

\subsubsection{Beta Diversity}

The fungal diversity of each plant tissue and rhizosphere soil was analyzed with a principal coordinates analysis (PCoA) and a cluster analysis. The results of PCoA based on the OTU level are shown in Figure 2. The first principal coordinate (PC1) in the sample from Hubei showed a representative contribution rate of $39.47 \%$ to the microbial fungi detected, and the representative contribution of the second principal coordinate (PC2) was $30.34 \%$ (Figure 2a). In the sample from Fujian, the representative contribution rate of PC1 to the microbial fungi detected was $45.15 \%$, and the representative contribution of PC2 was $24.99 \%$ (Figure $2 \mathrm{~b}$ ). The structures of the fungal communities in leaves and stems were similar between the samples from Hubei and Fujian. The structures of the fungal communities in the leaf and stem were clustered separately from those of the fungal communities in the roots and rhizosphere soil. In the cluster analysis (Figure 2c), the root, stem, and leaf samples from Hubei clustered together; however, the root samples were not an ingroup of the leaf + stem cluster, but a sister group of it. In the samples from Fujian (Figure 2d), the root and rhizosphere samples clustered together, and the stem and leaf samples clustered together.

\subsubsection{Composition of Endophytic and Rhizosphere Soil Fungi}

At the phylum level (Figure 3), Ascomycota was the dominant phylum in the Hubei samples, with relative abundances of $74.40-78.30 \%, 71.95-74.18 \%, 94.36-95.36 \%$ and $73.45-78.22 \%$, respectively, followed by Basidiomycota and Mortierellomycota. Ascomycota was also the dominant phylum in the Fujian samples. However, the relative abundances of the dominant flora in the Fujian rhizosphere soil samples differed significantly from those in the tissue samples. The relative abundances of Ascomycota, Mortierellomycota, and Basidiomycota were $31.63 \%, 39.55 \%$, and $15.73 \%$, respectively, in the rhizosphere soil; about $75 \%, 5 \%$, and $15 \%$, respectively, in the roots and leaves; and about $84.83 \%, 2.75 \%$, and $8.53 \%$, respectively, in the stem samples.

\subsection{Analysis of Endophytic Fungi Community Structure in H. serrata with Traditional Culture Methods}

In total, 15 and 13 strains were cultured from the root, stem, and leaf tissues of plants from Hubei and Fujian Provinces, respectively. The ITS rRNA gene sequence of strains was compared with the sequences deposited in the GenBank database (https: //blast.ncbi.nlm.nih.gov/Blast.cgi/ (accessed on 17 July 2020)). The species with the highest similarity was obtained and the results are shown in Table 1 . The phylogenetic tree was constructed by the neighbor-joining (NJ) method using the endophytic fungi isolated 
from Hubei and Fujian, and the species of these with the highest similarity. The preliminary identification of the endophytic fungi is given in Figure 4.

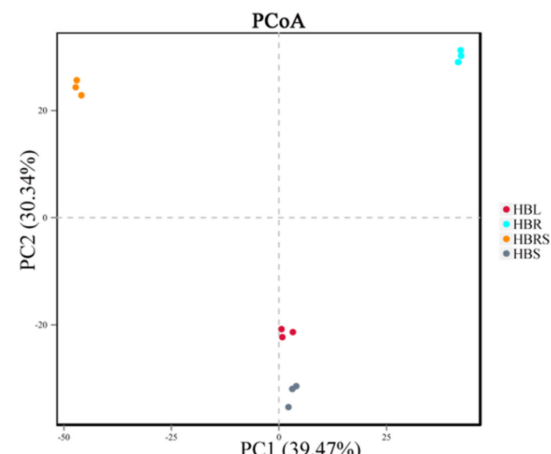

(a)

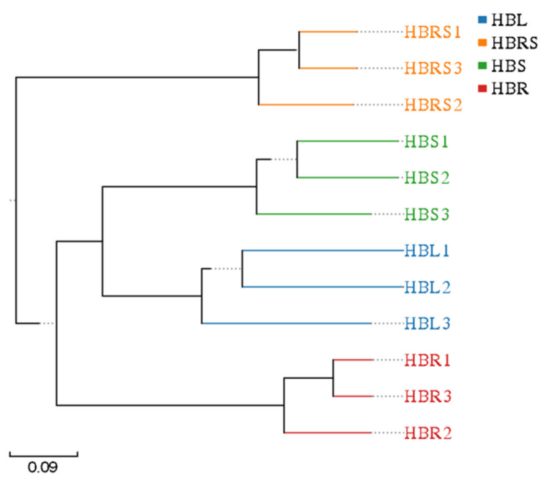

(c)

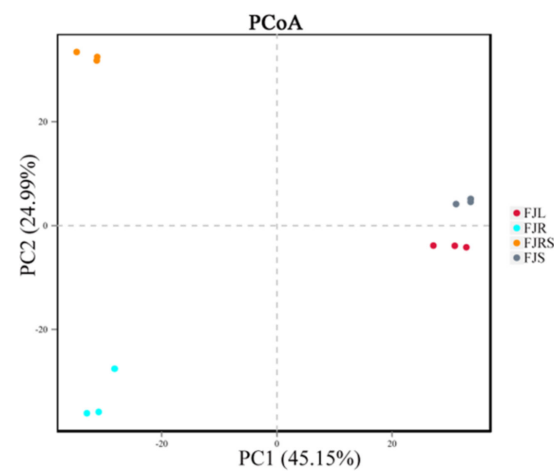

(b)

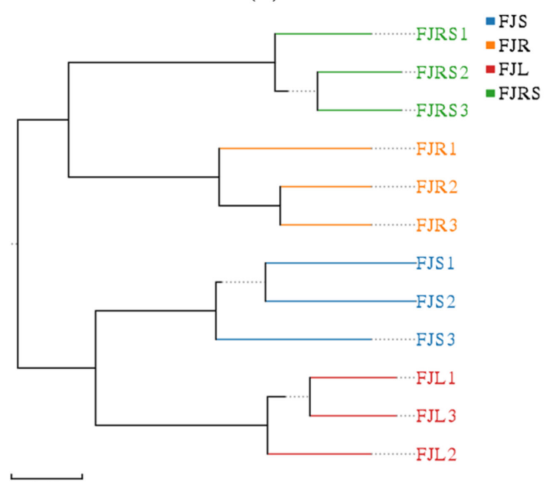

(d)

Figure 2. Principal coordinates analysis (a) Sample of Hubei Province. HBL: H. serrata leaf, HBR: H. serrata root, HBS: H. serrata stem, HBRS: rhizosphere soil; (b) Sample of Fujian Province. FJL: H. serrata leaf, FJR: $H$. serrata root, FJS: $H$. serrata stem, FJRS: rhizosphere soil) and cluster analysis (c) Sample of Hubei Province. HBL: H. serrata leaf, HBR: H. serrata root, HBS: H. serrata stem, HBRS: rhizosphere soil; (d) Sample of Fujian Province. FJL: H. serrata leaf, FJR: H. serrata root, FJS: H. serrata stem, FJRS: rhizosphere soil) of endophytic fungi in Huperzia serrata. The numbers 1, 2, and 3 in the names are used to indicate that the same tissue sample is repeated three times.

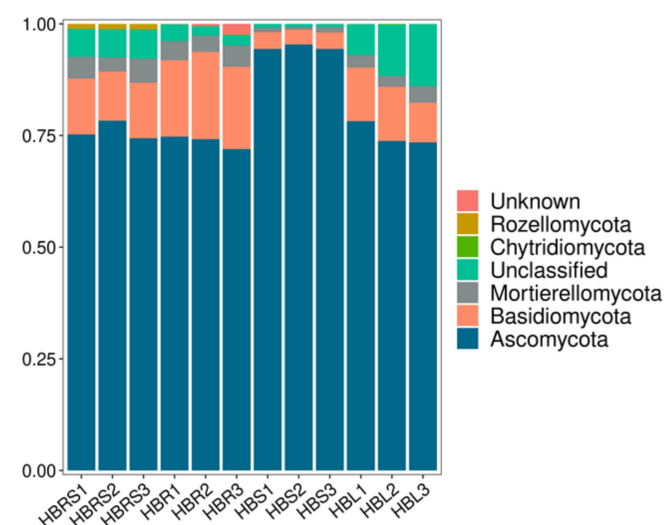

(a)

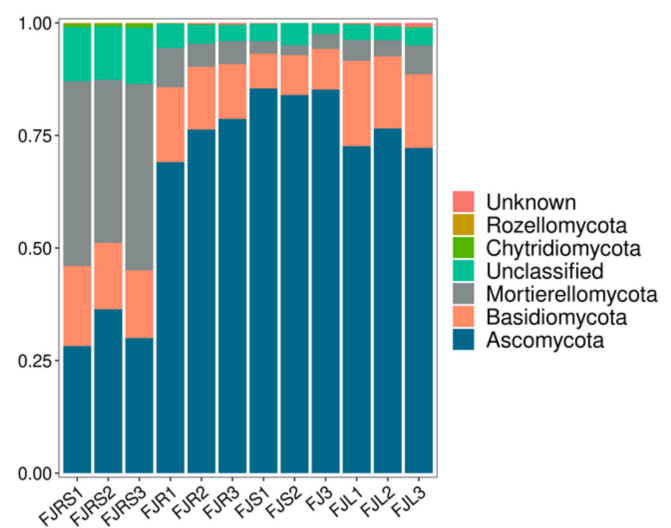

(b)

Figure 3. Fungal community composition at the phylum level. (a) Sample of Hubei Province. HBL: H. serrata leaf, HBR: H. serrata root, HBS: H. serrata stem, HBRS: rhizosphere soil; (b) Sample of Fujian Province. FJL: H. serrata leaf, FJR: H. serrata root, FJS: H. serrata stem, FJRS: rhizosphere soil). The numbers 1, 2, and 3 in the names are used to indicate that the same tissue sample is repeated three times. 
Table 1. Analysis of endophytic fungi isolated with traditional culture methods.

\begin{tabular}{|c|c|c|c|c|c|c|c|}
\hline Strains & $\begin{array}{l}\text { Closest Relatives } \\
\text { in NCBI }\end{array}$ & $\begin{array}{l}\text { Max. } \\
\text { Identity } \\
\%\end{array}$ & $\begin{array}{c}\text { AchE } \\
\text { Inhibitory } \\
\text { Activity (\%) }\end{array}$ & Strains & $\begin{array}{l}\text { Closest Relatives } \\
\text { in NCBI }\end{array}$ & $\begin{array}{l}\text { Max. } \\
\text { Identity } \\
\%\end{array}$ & $\begin{array}{c}\text { AchE } \\
\text { Inhibitory } \\
\text { Activity (\%) }\end{array}$ \\
\hline HBR-1 & Ascochyta caulina TF33 & 99.28 & $72.34 \pm 0.5$ & FJR-1 & Epicoccum nigrum UP_EPC81 & 99.81 & $41.24 \pm 1.5$ \\
\hline HBR-2 & $\begin{array}{c}\text { Cladosporium sphaerospermum } \\
\text { SCAU103 }\end{array}$ & 99.81 & $28.35 \pm 1.3$ & FJR-3 & Alternaria sp. strain AT-1 & 99.07 & $20.56 \pm 1.3$ \\
\hline HBR-3 & $\begin{array}{c}\text { Cladosporium cladosporioides } \\
\text { ZB18102902907 }\end{array}$ & 99.81 & $42.46 \pm 0.6$ & FJR-5 & $\begin{array}{l}\text { Arthrinium phaeospermum } \\
\text { OU-E } 38\end{array}$ & 96.92 & $35.23 \pm 0.7$ \\
\hline HBR-4 & Alternaria tenuissima RT29 & 99.26 & $36.29 \pm 0.6$ & FJR-6 & $\begin{array}{c}\text { Cladosporium cladosporioides } \\
\text { ZB18102902907 }\end{array}$ & 99.81 & $35.24 \pm 0.5$ \\
\hline HBS-1 & $\begin{array}{l}\text { Fusarium verticillioides } \\
\text { JCP2002 }\end{array}$ & 99.8 & $38.01 \pm 0.2$ & FJR-7 & Leptosphaeria sp. XJ23 & 99.64 & $5.61 \pm 0.3$ \\
\hline HBS-2 & $\begin{array}{c}\text { Alternaria sp. } \\
\text { OUCMBI101236 }\end{array}$ & 99.45 & $44.16 \pm 1.6$ & FJS-1 & Cladosporium sp. YS-134 & 99.61 & $33.23 \pm 0.9$ \\
\hline HBS-3 & Cladosporium sp. LQ122403 & 99.42 & $38.53 \pm 0.3$ & FJS-3 & $\begin{array}{l}\text { Aspergillus tabacinus } \\
\text { DUCC5716 }\end{array}$ & 99.45 & $26.36 \pm 1.1$ \\
\hline HBS-5 & $\begin{array}{l}\text { Arthrinium rasikravindrae } \\
\text { OUCMBI110096 }\end{array}$ & 99.65 & $23.49 \pm 2.1$ & FJS-5 & $\begin{array}{l}\text { Aspergillus tabacinus } \\
\text { 26R-5-F07 }\end{array}$ & 99.45 & $50.38 \pm 0.3$ \\
\hline HBS-6 & Coprinellus radians xsd 08234 & 95.41 & $17.92 \pm 0.8$ & FJL-1 & $\begin{array}{l}\text { Epicoccum nigrum } \\
\text { UP_EPC_51 }\end{array}$ & 99.04 & $10.3 \pm 0.7$ \\
\hline HBL-1 & Alternaria alternata MS18-2 & 99.45 & $50.32 \pm 1.8$ & FJL-2 & $\begin{array}{c}\text { Aspergillus sydowii strain } \\
\text { AsN19C03 }\end{array}$ & 99.44 & $29.78 \pm 1.9$ \\
\hline HBL-2 & $\begin{array}{l}\text { Cladosporium cladosporioides } \\
\text { C5 }\end{array}$ & 100 & $39.55 \pm 1.2$ & FJL-3 & Cladosporium sp. STE-U 5371 & 99.62 & $54.36 \pm 1.1$ \\
\hline HBL-3 & Alternaria sp. CDCF2534 & 99.81 & $1.66 \pm 0.6$ & FJL-4 & Alternaria tenuissima M9 & 98.89 & $39.79 \pm 0.4$ \\
\hline HBL-4 & $\begin{array}{c}\text { Cladosporium halotolerans } \\
\text { ENDO-PINE683- } \\
\text { BOTTOMA }\end{array}$ & 99.81 & $55.21 \pm 1.3$ & FJL-5 & Aspergillus sp. Z-Y-47 & 99.3 & $60.54 \pm 0.5$ \\
\hline HBL-6 & Alternaria sp. CDCF2530 & 99.63 & $52.26 \pm 1.1$ & & & & \\
\hline HBL-7 & $\begin{array}{l}\text { Cladosporium cladosporioides } \\
\text { ZJDF16 }\end{array}$ & 99.61 & $50.41 \pm 0.4$ & & & & \\
\hline
\end{tabular}

Note: Strains HBR-1, HBR-2, HBR-3 and HBR-4 were isolated from roots of H. serrata in Hubei Province; Strains HBS-1, HBS-2, HBS-3, HBS-5, HBS-6 were isolated from stems of $H$. serrata in Hubei Province; Strains HBL-1, HBL-2, HBL-3, HBL-4, HBL-6, HBL-7 were isolated from leaves of H. serrata in Hubei Province. Strains FJR-1, FJR-3, FJR-5, FJR-6 were isolated from roots of H. serrata in Fujian Province; Strains FJS-1, FJS-3, FJS-5 were isolated from stems of $H$. serrata in Fujian Province; Strains FJL-1, FJL-2, FJL-3, FJL-4, FJL-5 were isolated from leaves of $H$. serrata in Fujian Province. The inhibition effects of fungal extracts on AChE activity in vitro were based on three replicate tests. The inhibition effect of authentic HupA was $92.6 \pm 0.3$; values are mean $\pm \mathrm{SE}$ of three replications.

\subsection{Differences in Endophytic Fungi Detected with Traditional Culture Methods and High-Throughput DNA Sequencing}

\subsubsection{Differences in Endophytic Fungal Community Structures Detected}

When the endophytic fungi were isolated with traditional culture methods, two phyla, three classes, five orders, six families, and six genera were detected in the samples from Hubei, and one phylum, three classes, five orders, five families, and six genera were detected in the samples from Fujian. In contrast, high-throughput sequencing detected five phyla, 22 classes, 55 orders, 120 families, and 178 genera in the samples from Hubei and five phyla, 22 classes, 54 orders, 124 families, and 196 genera in the samples from Fujian (Table 2). These results indicate that traditional culture methods seriously underestimated the species composition of the endophytic fungi in H. serrata. More microbial species were detected with the high-throughput sequencing method than with the traditional culture method. High-throughput sequencing has obvious advantages over the pure culture method, and these advantages were greatest at these levels. 


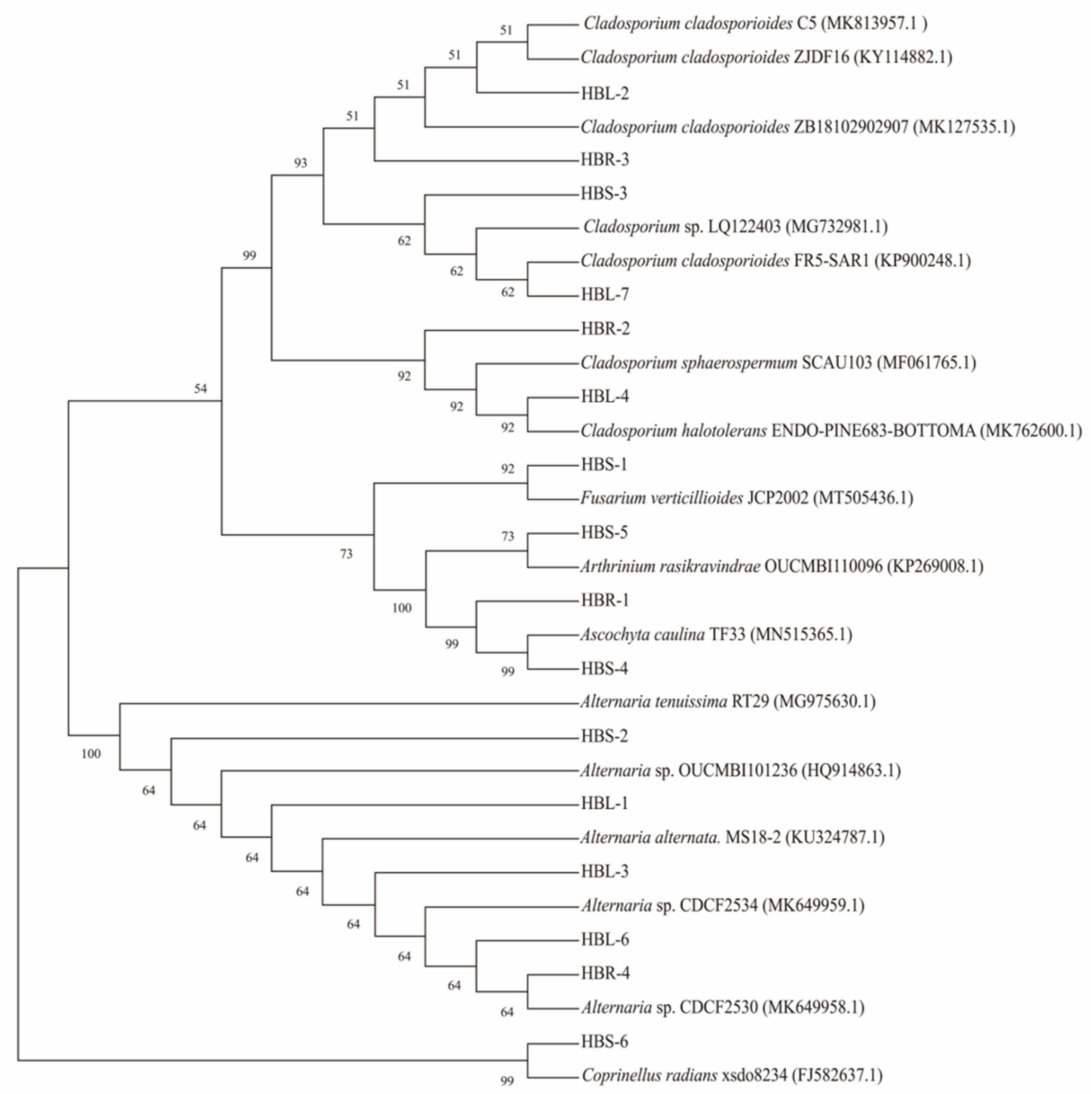

(a)

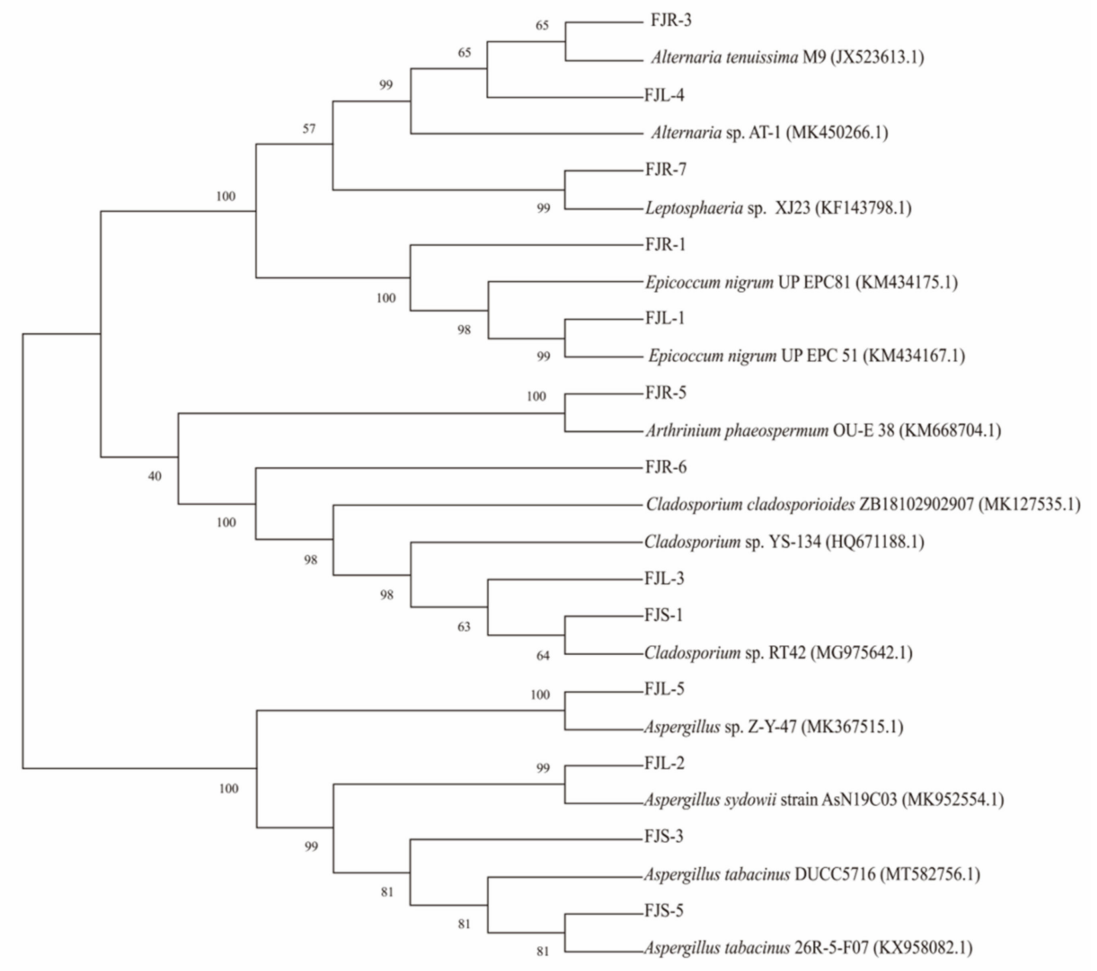

(b)

Figure 4. Neighbor-joining tree of the ITS sequences of endophytic fungi from Huperzia serrata. (a) phylogenetic tree was constructed based on fungi isolated from Huperzia serrata in Hubei province; (b) phylogenetic tree was constructed based on fungi isolated from Huperzia serrata in Fujian province). 
Table 2. Community structures of endophytic bacteria detected with traditional culture methods and high-throughput sequencing at each classification level.

\begin{tabular}{ccccccc}
\hline Sample & Items & Phylum & Class & Order & Family & Genus \\
\hline \multirow{2}{*}{ Hubei } & traditional culture methods & 2 & 3 & 5 & 6 & 6 \\
& high-throughput sequencing & 5 & 22 & 55 & 120 & 178 \\
\hline \multirow{2}{*}{ Fujian } & traditional culture methods & 1 & 3 & 5 & 5 & 6 \\
& high-throughput sequencing & 5 & 22 & 54 & 124 & 196 \\
\hline
\end{tabular}

\subsubsection{Differences in Endophytic Fungal Diversity}

The microbial diversity indices of the endophytic fungi in $H$. serrate were analyzed. The Shannon indices for the endophytic fungi of H. serrata isolated with traditional culture methods were 2.099 in Hubei and 2.416 Fujian, whereas the Simpson indices were 0.711 and 0.793 , respectively. However, the corresponding Shannon indices calculated from highthroughput sequencing data were 3.426 and 3.939, respectively, and the corresponding Simpson indices were 0.828 and 0.936 , respectively. These findings show that the diversity of endophytic fungi detected with high-throughput sequencing was higher than that detected with traditional culture methods. They also show that the endophytic fungus community's structure in $H$. serrata is complex and stable.

\subsection{Acetylcholine-Inhibiting Activity of Endophytic Fungi Isolated with Traditional Culture Methods}

The in vitro AChE inhibitory activity of endophytic fungal extracts from two Chinese provinces were tested (Table 1). Among the strains isolated with traditional culture methods, those with high AChE inhibitory activity (>50\% inhibition) accounted for $33.33 \%$ and $23.08 \%$ in the samples from Hubei and Fujian, respectively; those with moderate activity (30-50\% inhibition) for $40 \%$ and $38.46 \%$, respectively; and those with low activity ( $<30 \%$ inhibition) for $26.67 \%$ and $38.46 \%$, respectively. The endophytic fungal sample HBR-1 from Hubei and sample FJL-5 from Fujian had the strongest inhibitory effects on AChE, with inhibition rates of $72.34 \%$ and $60.54 \%$, respectively.

\section{Discussion}

In this study, traditional culture methods and high-throughput sequencing were used to study the diversity of endophytic fungi in H. serrata from Hubei and Fujian. Both methods showed that these endophytic fungi were highly diverse. Compared with highthroughput sequencing, the traditional culture methods identified relatively fewer cultures, which may be attributable to the influence of the culture conditions, which limited the numbers of microorganisms obtained.

When the different tissues of the plants from Hubei and Fujian were processed with traditional culture methods, 15 and 13 endophytic fungi were isolated, respectively (Table 1). The endophytes in both places were predominantly from the phylum Ascomycota, except a unique strain from Basidiomycota in the Hubei sample, but the distribution was different at the genus level. Both the Hubei and Fujian samples contained Alternaria, Arthrinium, and Cladosporium. Ascochyta, Fusarium and Coprinellus were only isolated from the Hubei sample and Epicoccum, Leptosphaeria, and Aspergillus from the Fujian sample. These genera have also been isolated in previous studies [21,30-33]. The results show some differences in the diversity of endophytic fungi at different geographic locations. Wang et al. studied the composition of endophytic fungi of $H$. serrata from Lushan Mountain and Jinggang Mountain, and also found that there were many differences, indicating that geographical environment plays a more important role in affecting the diversity of endophytic fungi, even if from the same plant $[21,33]$. Plants in different habitats provide different environmental and nutritional conditions for the microorganisms within them, and these conditions affect the complex symbiotic relationships between the microorganisms and plants, generating different endophytic flora. When isolating and culturing the endophytic 
fungi of $H$. serrata, the natural ecological environment in which the plant grows must be simulated as closely as possible to maximize the endophytic fungal resources available and better reflect the natural endophytic fungal diversity of $H$. serrata. Because we cannot completely simulate this natural environment, fewer endophytic fungi were isolated in the laboratory. During the process of isolation and culturing in the laboratory, the first thing to be considered is the interaction between endophytes and hosts. The second may be the interconnection among endophytes [34]. We realize that the strategies of culturing "unculturable microorganisms" have attracted widespread attention. Mu et al. concluded that simulating the natural environment is an effective strategy to isolate active bacteria $[35,36]$.

High-throughput sequencing detected Ascomycota, Basidiomycota, Mortierellomycota, Chytridiomycota, and Rozellomycota in the H. serrata plants from both Hubei and Fujian Provinces. The dominant phylum was Ascomycota, followed by Basidiomycota and Mortierellomycota; therefore, the composition of endophytic fungi at the phylum level was similar, to a certain extent, in plants under different growth and environmental conditions, but the relative abundances of the various phyla differed in response to the different environments for plant growth. At the genus level, the traditional separation methods detected only six different strains, far fewer than were detected with high-throughput sequencing. All the genera detected with traditional culture methods were also detected with highthroughput sequencing (Supplementary Materials Tables S1 and S2), except Ascochyta. This exception may be attributable to its missed detection with high-throughput sequencing. These results combined with the $\alpha$ diversity indices analysis show that high-throughput sequencing better reflects the distribution of endophytic fungi in $H$. serrate than traditional culture methods. The abundance, uniformity, and diversity of OTUs in the roots, stems, leaves, and rhizosphere soil were evaluated in terms of their alpha diversity. The OTU richness in the rhizosphere clearly differed from that in the plant roots, stems, and leaves, and the rhizosphere microbial richness in the two provinces was significantly higher than the richness of the plant endophytic flora. However, there were no significant differences in the OTU abundances among the different tissues. Our results differ from the results of Beckers et al. [37]. Their research showed that the endophytic bacteria in the rhizosphere soil, root, and stem had clearly distinguishable OTU abundances. This may be related to the different plant species and microflora types studied. To further compare the structures of fungal flora in the plant rhizosphere soil and in different plant tissues, all the samples were clustered with PCoA and cluster analysis. All the fungal samples were clustered according to the different plant tissues, and the result indicated that endophytic fungi differ across different tissues. The cluster analysis showed that the endophytic fungi in Fujian showed similar clustering to that of bacteria [37]. The endophytic fungi of Fujian samples in the rhizosphere soil and root tissues clustered together, and the stem and leaf tissues clustered together. These findings support the idea that rhizosphere sediments and the root exudates of the host plant enhance the richness of the microbial community in the rhizosphere soil [38,39]. The microorganisms in the rhizosphere soil travel through the endoderm and root stele to the xylem vessels, ultimately colonizing the whole plant [40]. However, the clustering of the endophytic fungi in the rhizosphere soil and the plant tissues in the Hubei region, where the endophytic fungi from the roots, stems, and leaves clustered together, differed from that in Fujian Province. This difference may be related to the different environments of plant growth in these two places. The soil in which the plants grew in Fujian was relatively moist, which may have promoted the movement of the rhizosphere soil fungi into the plants to colonize them. Several studies have shown that the structures of fungal communities can vary according to the soil type, plant compartment, plant species, and season [41-43].

The endophytic fungi of $H$. serrata in the Hubei and Fujian Provinces were separated and purified with traditional culture methods. The endophytic fungi HBR-1 and FJL-5 from Hubei and Fujian, respectively, showed the best inhibitory effects on AChE (inhibition rate $>50 \%$ ), with inhibition rates of $72.34 \%$ and $60.54 \%$, respectively. This indicates that the production and development of AchE inhibitors by endophytic fungi have certain 
potential. A statistical analysis of the fungi with higher AChE inhibitory activity (inhibition rates $>50 \%$ ) indicated that most of the strains with high AChE inhibitory activity were isolated from $\mathrm{H}$. serrata leaf tissue, which accounted for $80 \%$ and $66.67 \%$ of the high enzyme producers from Hubei and Fujian, respectively. Cui et al. analyzed the content of HupA in the endophytic fungi community and the species of HupA, and found that most of the endophytic fungi that showed a significant positive correlation with the acetylcholinesterase inhibitor HupA were isolated from leaves [44]. This phenomenon may be related to the higher content of the AChE inhibitor in the leaves of $H$. serrata plants than in the stems or roots [45]. It also supports the theory of Young et al. that, through evolution, symbiotic endophytes have developed mechanisms for the biosynthesis and tolerance of high levels of secondary metabolites, so as to better compete with and survive in the tissues of medicinal plants [46].

The results of the high-throughput sequencing method more comprehensively reflect the diversity of endophytic fungi in $\mathrm{H}$. serrata than those of the traditional culture methods, which has major significance for research into endophytic fungi. The isolation of endophytic fungal resources with traditional culture methods also has valuable applications, especially because some of these fungi have antibacterial and growth-promoting activities. A variety of metabolites can be isolated from these endophytic fungi, which have broad potential utility in the development and utilization of natural products. The combination of high-throughput sequencing and traditional pure culture methods reflects the diversity of the endophytic fungi of $H$. serrata most comprehensively, and also allows their targeted isolation and purification, the isolation of greater numbers of endophytic fungi, the enrichment of the endophytic fungal resource pool, and the provision of materials for research. However, further research is needed. Furthermore, the use of degenerated primer pairs for genes involved in the biosynthesis of the AchE inhibitor, in order to assess the diversity of fungi able to produce this compound, is still worth exploring $[47,48]$.

Supplementary Materials: The following are available online at https:/ /www.mdpi.com/article/10 $.3390 /$ su132112073/s1, Table S1. Community structure of endophytic bacteria in Hubei at the genus level, detected with high-throughput sequencing. Table S2. Community structure of endophytic bacteria in Fujian at the genus level, detected with high-throughput sequencing.

Author Contributions: Conceptualization, project administration, resources, Funding acquisition, Supervision H.Y.; writing—original draft preparation, Z.L.; writing—review and editing, Y.M.; supervision, D.Z.; visualization, L.X.; investigation, Methodology, Z.L. and Y.M. All authors have read and agreed to the published version of the manuscript.

Funding: This research was funded by Natural Science Foundation of Jiangxi Province, grant number 20181BAB214003, and funded by Project of Graduate Innovation Foundation from Education Department of Jiangxi Province, grant number YC2020-S184.

Institutional Review Board Statement: Not applicable.

Informed Consent Statement: Not applicable.

Data Availability Statement: The data presented in this study are openly available in National Center for Bio-technology Information (NCBI) database, BioProject accession number PRJNA655213 and Sequence Read Archive (SRA) accession numbers SRR12401455-SRR12401478.

Conflicts of Interest: The authors declare no conflict of interest.

\section{References}

1. Prieto, J.A.F.; Aguiar, C.; Dias, E.; Casado, M.D.L.A.F.; Homet, J. The genus Huperzia (Lycopodiaceae) in the Azores and Madeira. Bot. J. Linn. Soc. 2008, 158, 522-533. [CrossRef]

2. Yang, Q.P.; Kou, X.L.; Fugal, K.B.; McLaughlin, J.L. Determination of huperzine A in formulated products by reversed-phaseliquid chromatography using diode array and electrospray ionization mass spectrometric detection. Phytomedicine 2003, 10, 200-205. [CrossRef]

3. Chen, X.Y.; Qi, Y.D.; Wei, J.H.; Zhang, Z.; Wang, D.L.; Feng, J.D.; Gan, B.C. Molecular identification of endophytic fungi from medicinal plant Huperzia serrata based on rDNA ITS analysis. World J. Microbiol. Biotechnol. 2011, 27, 495-503. [CrossRef] 
4. Ma, X.Q.; Tan, C.H.; Zhu, D.Y.; Gang, D.R.; Xiao, P.G. Huperzine A from Huperzia species-An ethnopharmacolgical review. J. Ethnopharmacol. 2007, 113, 15-34. [CrossRef] [PubMed]

5. Liu, Y.C.; Zhang, Z.J.; Su, J.; Peng, L.Y.; Pan, L.T.; Wu, X.D.; Zhao, Q.S. Lycodine-Type Lycopodium Alkaloids from the Whole Plants of Huperzia serrata. Nat. Prod. Bioprospect. 2017, 7, 405-411. [CrossRef]

6. Wu, J.G.; Wang, Y.Y.; Zhang, Z.L.; Yu, B. Herbal medicine in the treatment of Alzheimer's disease. Chin. J. Integr. Med. 2015, 2, 102-107. [CrossRef] [PubMed]

7. Rasic, J.S.; Ivanovic, N.D.; Andjelkovic, M.S.; Nedeljkovic, I.P.; Nikolic, I.R.; Stojanovic, S.D.; Ristic-Medic, D.K.; Takic, M.M.; Djordjevic, B.I.; Dikic, N.V. Influence of Higenamine on Exercise Performance of Recreational Female Athletes: A Randomized Double-Blinded Placebo-Controlled Trial. Front. Psychol. 2021, 12. [CrossRef]

8. Petrini, O. Fungal Endophytes of Tree Leaves. In Microbial Ecology of Leaves; Andrews, J.H., Hirano, S.S., Eds.; Springer: New York, NY, USA, 1991; pp. 179-197.

9. Saikkonen, K.; Faeth, S.H.; Helander, M.; Sullivan, T.J. Fungal endophytes: A continuum of interactions with host plants. Annu. Rev. Ecol. Syst. 1998, 29, 319-343. [CrossRef]

10. Li, J.; Zhao, J.L.; Xu, L.J.; Zhou, L.G.; Li, X.; Wang, J.G. Endophytic fungi from rhizomes of Paris polyphylla var. yunnanensis. World J. Microbiol. Biotechnol. 2008, 24, 733-737. [CrossRef]

11. Reiter, B.; Sessitsch, A. Bacterial endophytes of the wildflower Crocus albiflorus analyzed by characterization of isolates and by a cultivation-independent approach. Can. J. Microbiol. 2006, 52, 140-149. [CrossRef]

12. Compant, S.; Duffy, B.; Nowak, J.; Clément, C.; Barka, E.A. Use of Plant Growth-Promoting Bacteria for Biocontrol of Plant Diseases: Principles, Mechanisms of Action, and Future Prospects. Appl. Environ. Microbiol. 2005, 71, 4951-4959. [CrossRef] [PubMed]

13. Frankowski, J.; Lorito, M.; Scala, F.; Schmid, R.; Berg, G.; Bahl, H. Purification and properties of two chitinolytic enzymes of Serratia plymuthica HRO-C48. Arch. Microbiol. 2001, 176, 421-426. [CrossRef] [PubMed]

14. Ezra, D.; Castillo, U.F.; Strobel, G.A.; Hess, W.M.; Porter, H.; Jensen, J.B.; Condron, M.A.M.; Teplow, D.; Sears, J.; Maranta, M.; et al. Coronamycins, peptide antibiotics produced by a verticillate Streptomyces sp. (MSU-2110) endophytic on Monstera sp. Microbiology 2004, 150, 785-793. [CrossRef]

15. Aken, B.V.; Peres, C.M.; Doty, S.L.; Yoon, J.M.; Schnoor, J.L. Methylobacterium populi sp. nov. a novel aerobic, pink-pigmented, facultatively methylotrophic, methane-utilizing bacterium isolated from poplar trees (Populus deltoidesxnigra DN34). Int. J. Syst. Evol. Microbiol. 2004, 54, 1191-1196. [CrossRef] [PubMed]

16. Aly, A.H.; Debbab, A.; Kjer, J.; Proksch, P. Fungal endophytes from higher plants: A prolific source of phytochemicals and other bioactive natural products. Fungal Divers 2010, 41, 1-16. [CrossRef]

17. Stierle, A.; Strobel, G.; Stierle, D. Taxol and taxane production by Taxomyces andreanae, an endophytic fungus of Pacific yew. Science 1993, 260, 214. [CrossRef] [PubMed]

18. Strobel, G.; Daisy, B.; Castillo, U.; Harper, J. Natural products from endophytic microorganisms. J. Nat. Prod. 2004, 67, 257-268. [CrossRef]

19. Strobel, G.; Daisy, B. Bioprospecting for microbial endophytes and their natural products. Microbiol. Mol. Biol. Rev. 2003, 67, 491-502. [CrossRef]

20. Han, W.X.; Han, Z.W.; Jia, M.; Zhang, H.; Li, W.Z.; Yang, L.B.; Liang, F.; Han, L. Zhao, N.; Li, X.F. Five novel and highly efficient endophytic fungi isolated from Huperzia serrata expressing huperzine A for the treatment of Alzheimer's disease. Appl. Microbiol. Biotechnol. 2020, 104, 9159-9177. [CrossRef]

21. Wang, Y.; Zeng, G.Q.; Zhang, Z.B.; Yan, R.M.; Wang, L.Y.; Zhu, D. Isolation and characterization of endophytic huperzine A-producing fungi from Huperzia serrata. J. Ind. Microbiol. Biotechnol. 2011, 38, 1267-1278. [CrossRef]

22. Beckers, B.; Michiel, O.D.B.; Weyens, N.; Acker, R.V.; Montagu, M.V.; Boerjan, W.; Vangronsveld, J. Lignin engineering in field-grown poplar trees affects the endosphere bacterial microbiome. Proc. Natl. Acad. Sci. USA 2016, 113, 2312. [CrossRef] [PubMed]

23. Mcinroy, J.A.; Kloepper, J.W. Survey of indigenous bacterial endophytes from cotton and sweet corn. Plant Soil 1995, 173, 337-342. [CrossRef]

24. Bokulich, N.A.; Mills, D.A. Improved Selection of Internal Transcribed Spacer-Specific Primers Enables Quantitative, Ultra-HighThroughput Profiling of Fungal Communities. Appl. Environ. Microbiol. 2013, 79, 2519-2526. [CrossRef] [PubMed]

25. Edgar, R.C. Search and clustering orders of magnitude faster than BLAST. Bioinformatics 2010, 26, 2460-2461. [CrossRef] [PubMed]

26. Caporaso, J.G.; Kuczynski, J.; Stombaugh, J.; Bittinger, K.; Bushman, F.D.; Costello, E.K.; Fierer, N.; Peña, A.G.; Goodrich, J.K.; Gordon, J.I.; et al. QIIME allows analysis of high-throughput community sequencing data. Nat. Methods 2010, 7, 335-336. [CrossRef]

27. Tamura, K.; Stecher, G.; Peterson, D.; Filipski, A.; Kumar, S. MEGA6: Molecular Evolutionary Genetics Analysis Version 6.0. Mol. Biol. Evol. 2013, 30, 2725-2729. [CrossRef] [PubMed]

28. SzypulA, W.; Pietrosiuk, A.; Suchocki, P.; Olszowska, O.; Furmanowa, M.; Kazimierska, O. Somatic embryogenesis and in vitro culture of Huperzia selago shoots as a potential source of huperzine A. Plant Sci. 2005, 168, 1443-1452. [CrossRef]

29. Rahman, A.U.; Choudhary, M.I.; Thomsen, W.J. Bioassay Techniques for Drug Development, 1st ed.; CRC Press: Florida, FL, USA, 2001. 
30. Le, T.T.M.; Hoang, A.T.H.; Le, T.T.B.; Vo, T.T.B.; Quyen, D.V.; Chu, H.H. Isolation of endophytic fungi and screening of Huperzine A-producing fungus from Huperzia serrata in Vietnam. Sci. Rep. 2019, 9, 16152. [CrossRef]

31. Yang, H.Y.; Qi, B.W.; Ding, N.; Jiang, F.F.; Jia, F.F.; Luo, Y.; Xu, X.P.; Wang, L.L.; Zhu, Z.X.; Liu, X.; et al. Polyketides from Alternaria alternata MT-47, an endophytic fungus isolated from Huperzia serrata. Fitoterapia 2019, 137, 104282. [CrossRef]

32. Zhang, Z.B.; Zeng, Q.G.; Yan, R.M.; Wang, Y.; Zou, Z.R.; Zhu, D. Endophytic fungus Cladosporium cladosporioides LF70 from Huperzia serrata produces Huperzine A. World J. Microbiol. Biotechnol. 2011, 27, 479-486. [CrossRef]

33. Wang, Y.; Lai, Z.; Li, X.X.; Yan, R.M.; Zhang, Z.B.; Yang, H.L.; Zhu, D. Isolation, diversity and acetylcholinesterase inhibitory activity of the culturable endophytic fungi harboured in Huperzia serrata from Jinggang Mountain, China. World J. Microb. Biot. 2016, 32. [CrossRef] [PubMed]

34. Peng, L.L.; Wang, Q.; Xin, M.X. Advanced in uncultivable microorgnisms in nature. J. Microbiol. 2011, 31, 75-79. [CrossRef]

35. Mu, D.S.; Ouyang, Y.; Chen, G.J.; Du, Z.J. Strategies for culturing active/dormant marine microbes. Marine Life Sci. Technol. 2020, 3, 121-131. [CrossRef]

36. Kaeberlein, T.; Lewis, K.; Epstein, S.S. Isolating “Uncultivable” Microorganisms in Pure Culture in a Simulated Natural Environment. Science 2002, 296, 1127-1129. [CrossRef]

37. Beckers, B.; Beeck, M.O.D.; Weyens, N.; Boerjan, W.; Vangronsveld, J. Structural variability and niche differentiation in the rhizosphere and endosphere bacterial microbiome of field-grown poplar trees. Microbiome 2017, 5, 25. [CrossRef] [PubMed]

38. Lugtenberg, B.; Kamilova, F. Plant-growth-promoting rhizobacteria. Annu. Rev. Microbiol. 2009, 63, 541-556. [CrossRef]

39. Bais, H.P.; Weir, T.L.; Perry, L.G.; Gilroy, S.; Vivanco, J.M. The role of root exudates in rhizosphere interactions with plants and other organisms. Annu. Rev. Plant Biol. 2006, 57, 233-266. [CrossRef] [PubMed]

40. Compant, S.; Clément, C.; Sessitsch, A. Plant growth-promoting bacteria in the rhizo- and endosphere of plants: Their role, colonization, mechanisms involved and prospects for utilization. Soil Biol. Biochem. 2009, 42, 669-678. [CrossRef]

41. Devin, C.D.; Damaris, D.; Citlali, F.G.; Stephen, G.; Scott, C.; Tanja, W.; North, G.; Visel, A.; Partida-Martinez, L.P.; Tringe, S.G. Plant compartment and biogeography affect microbiome composition in cultivated and native Agave species. New Phytol. 2016, 209, 798-811. [CrossRef]

42. Aurore, C.; Tristan, C.; Lengellé, J.; Defossez, E.; Vacher, C.; Robin, C.; Buée, M.; Marcais, B. Leaf and Root-Associated Fungal Assemblages Do Not Follow Similar Elevational Diversity Patterns. PLoS ONE 2014, 9, e100668. [CrossRef]

43. Shakya, M.; Gottel, N.; Castro, H.F.; Yang, Z.K.; Gunter, L.; Labbé, J.; Muchero, W.; Bonito, G.M.; Vilgalys, R.; Tuskan, G.; et al. A Multifactor Analysis of Fungal and Bacterial Community Structure in the Root Microbiome of Mature Populus deltoides Trees. PLoS ONE 2013, 8, e76382. [CrossRef] [PubMed]

44. Cui, L.L.; Noushahi, H.A.; Zhang, Y.P.; Liu, J.X.; Cosoveanu, A.; Liu, Y.; Yan, L.; Zhang, J.; Shu, S.H. Endophytic Fungal Community of Huperzia serrata: Diversity and Relevance to the Production of Huperzine A by the Plant Host. Molecules 2021, 26, 892. [CrossRef] [PubMed]

45. Yuan, J.Q.; Zhou, X.L.; Wang, S.; Yan, Z.G.; Feng, S.X.; Jiang, J.M.; Zhu, D.Y.; Ma, X.J. Simultaneous determination of huperzine A and huperzine B in different parts of Huperzia serrata from different habitats by HPLC. J. Pharm. Anal. 2012, 32, 1541-1544. [CrossRef]

46. Young, D.H.; Michelotti, E.L.; Swindell, C.S.; Krauss, N.E. Antifungal properties of taxol and various analogues. Experientia 1992, 48, 882-885. [CrossRef] [PubMed]

47. Yang, M.Q.; Wu, S.W.; You, W.J.; Jaisi, A.; Xiao, Y.L. Selection of Reference Genes for Expression Analysis in Chinese Medicinal Herb Huperzia serrata. Front. Pharmacol. 2019, 10, 44. [CrossRef] [PubMed]

48. Yang, G.D.; Li, P.; Meng, L.F.; Xv, K.Y.; Dong, F.M.; Qiu, Y.; He, L.; Lin, L. Diversity and communities of culturable endophytic fungi from different tree peonies (geoherbs and non-geoherbs), and their biosynthetic potential analysis. Braz. J. Microbiol. 2018, 49, 47-58. [CrossRef] 